\title{
Optimal Price Discount Policy for Non-instantaneous Deteriorating Items with Stock-dependent and Time Decreasing Demand
}

\author{
Bindu Vaish \\ D. N. College, Meerut, India \\ E-mail: bindu58@rediffmail.com \\ Garima Garg \\ RKGIT, Ghaziabad \\ E-mail: garima6982@gmail.com
}

Received: January 30, 2011 Accepted: February 28, 2011 doi:10.5539/jmr.v3n3p119

\begin{abstract}
This paper deals with an inventory model with stock dependent and time varying decreasing demand which increases under the effect of price discount. After some time before deterioration starts a fixed discount on unit selling price is given and when product starts to deteriorate the demand becomes stock independent and is only time decreasing. In such a situation another discount on the selling price is given to boost up the demand and to reduce the loss due to deterioration. The model determines optimal discount to be given on unit selling price during deterioration so as to maximize the total profit. Numerical examples are presented to illustrate the model and sensitivity analysis is also reported.
\end{abstract}

Keywords: Non-instantaneous deterioration, Stock and time dependent demand, Price discount

\section{Introduction}

In most of the existing inventory models in the literature it is assumed that items can be stored indefinitely to meet the future demands. However, certain types of commodities either deteriorate or become obsolete in the course of time and hence are unstable. Thus it is important to control and maintain the inventories of deteriorating items for the modern corporation. The first attempt to describe the optimal ordering policies for deteriorating items was made by Ghare \& Schrader (1963). They presented an EOQ model for an exponentially decaying inventory. Later Nahmias (1982), Cobbarert \& Oudheusden (1996), Chung et al. (2000), Goyal \& Giri (2001) provided an excellent \& detailed review of deteriorating inventory literatures.

In the existing literature, in maximum inventory models for deteriorating items it is assumed that the deterioration occurs as soon as the commodities arrive in inventory. However, in real life, most goods would have a span of maintaining quality or original condition, i.e. during that period no deterioration occurs. This phenomenon is termed as 'non-instantaneous deterioration', given by Wu et al. (2006). This type of phenomena can be commonly observed in food stuffs, fruits, green vegetables and fashionable goods, which have a span of maintaining fresh quality \& during that period there is almost no spoilage and after some time some of the items will start to decay. For these kinds of items the assumption that the deterioration starts from the instant of arrival in stock may cause retailers to make inappropriate replenishment policy due to overvalue of the total annual relevant inventory cost. Thus it is necessary to consider the inventory problems for non-instantaneous deteriorating items. Lin and Shi (1999) classified inventory models into two categories decay models \& finite lifetime models. Castro \& Alfa (2004) proposed a lifetime replacement policy in discrete time for a single unit system. Ouyang et al. (2006) developed an inventory model for non-instantaneous deteriorating items with permissible delay in payments. Chang et al. (2010) developed optimal replenishment policies for non-instantaneous deteriorating items with stock-dependent demand.

In the existing literature of inventory models, the assumption of constant demand rate is not applicable to most of the products. The demand rate of any product is always in a dynamic state i.e. products experience fluctuations in the demand rate. Demand of goods may vary with time or with price or even with the instantaneous level of inventory displayed. Many contributions have been made to the replenishment problem when there is a linear increasing trend in demand within a time horizon, which is more realistic than the case where demand is constant. In real life, the demand of some existing products decreases with time when new products are launched. Thus the demand rate is decreasing with time for some products. Further the large amount of stock on display has a motivational effect on demand rate and increases the demand rate for a short span. Smith (1977) presented an inventory model where the demand rate is decreasing negativeexponentially. Benkherouf (1995) developed an inventory model for deteriorating items with decreasing time-varying demand \& shortages. Zhao et al. (2001) proposed a replenishment policy when the trend in demand is decreasing. Zhou (2003) presented a multi-warehouse inventory models for items with demand rate as a function of time which increases at a decreasing rate. Balkhi \& Benkherouf (2004) came up with an inventory model for deteriorating items with stock 
dependent \& time varying demand rate for a finite planning horizon. Yang et al. (2004) presented a replenishment policy with non-linear decreasing demand. Omar (2009) considered a production policy under linearly decreasing demand. Banerjee \& Sharma (2010) developed inventory models with price and time dependent seasonal demand.

It is a common practice for a supplier to offer discounts to customers. The motivation for offering discounts stems from the fact that it encourages the customers to purchase a large lot size than the regular one. From the supplier's perspective, he offer's discount to increase cash flow, to decrease the levels of inventory to reduce loss due to deterioration, to boost market share or simply to retain customers. Supplier has to decide the optimal discount and the optimal time when the discount is to be given to the customers. This is because the selling price should not be so high that it puts off the customers and not so low that the supplier losses out on the profits. Thus price discount is one of the key factors which influence demand and its effect cannot be ignored. Ardalan (1994) developed an inventory policy where temporary price discounts resulted in increase in demand. Wee \& Yu (1997) proposed an inventory model for deteriorating items with a temporary price discount. Papachristos \& Skouri (2003) presented an inventory models for deteriorating items where demand rate is a convex decreasing function of the selling price. Sana \& Chaudhuri (2008) presented an EOQ model with delay in payments and price discount offers. Hsu \& Yu (2009) proposed an EOQ model for imperfective items under a one-time-only discount. Panda et al. (2009) developed an EOQ model for perishable products with discounted selling price and stock dependent demand. Cardanas-Barron et al. (2010) presented an inventory model for determining the optimal ordering policies to take advantage of a one-time discount offer with allowed back orders.

The present paper is an extension of Panda et al. (2009). They proposed an EOQ model for an infinite time horizon for perishable products with discounted selling price and stock dependent demand with non-instantaneous constant rate of deterioration. In the present paper an inventory model is considered with stock dependent and time varying decreasing demand. Deterioration is non-instantaneous and time dependent. A temporary discount on selling price before the start of deterioration is given to enhance the demand in order to boost the inventory depletion rate. After some time when product starts deteriorating the demand becomes stock independent and is only time decreasing. In such a situation another discount on selling price is given to boost up the demand, to decrease the levels of inventory, so as to reduce loss due to deterioration. The model is developed for finite planning horizon. Numerical examples are provided to illustrate the optimization procedure. In addition the sensitivity analysis of the optimal solution with respect to parameters of the system is carried out.

\section{Assumptions and Notation}

\subsection{Assumptions}

The following assumptions are adopted:

The replenishment is instantaneous and lead time is zero.

The system operates for a prescribed period of a planning horizon.

Shortages are not allowed.

The deterioration rate is time dependent and the deterioration of items begins after a time $\mu$ from the instant of the arrival of the stock.

$I(t)$ is the inventory level at time $t$.

In each cycle the demand rate follows the pattern $D(t)=a+b I(t)-c t$ i.e. the demand is stock dependent and decreasing with time from the time of fresh replenishment up to the time $\mu$, and when deterioration starts at time $\mu$ it is only time decreasing i.e. $D(t)=a-c t$ where $a$ is a positive constant and $b$ and care the scale parameters where $a$ is a positive constant and $b$ and $c$ are the scale parameters where $0 \leq b, c \leq 1$.

$d_{1}\left(0 \leq d_{1} \leq 1\right)$ is the percentage discount offer on unit selling price before deterioration. $\alpha_{1}=\left(1-d_{1}\right)^{-n_{1}}\left(n_{1} \in R\right.$, the set of real numbers), is the effect of discounted selling price on demand before deterioration. When $d_{1} \rightarrow 0, \alpha_{1} \rightarrow 1$ i.e. for no pre-deterioration discount the demand is assumed to be $a+b I(t)-c t . d_{2}\left(0 \leq d_{2} \leq 1\right)$ is the percentage discount offer on unit selling price during deterioration. $\alpha_{2}=\left(1-d_{2}\right)^{-n_{2}}\left(n_{2} \in R\right.$, the set of real numbers), is the effect of discounted selling price on demand during deterioration. When $d_{2} \rightarrow 0, \alpha_{2} \rightarrow 1$ i.e. the demand of decreased quality items remains same.

In day to day life it is commonly observed that the demand of fresh goods is increased significantly if a discount is offered on selling price and the demand of decreased quality items is also increased if a discount is offered on selling price. Since the demand is boosted if the discounts are offered therefore it is concluded that the demand is partially stock and time dependent and partially stock, time and selling price dependent if the discounts are given.

\subsection{Notations}

The following notations are used in the model:

$\mathrm{A}$ is the set up cost. 
$C_{1}$ is the unit holding cost per item.

$s$ is the unit selling price of the product per unit.

$d_{1}$ is the percentage discount offer per unit before deterioration.

$d_{2}$ is the percentage discount offer per unit after deterioration.

$C_{4}$ is the unit purchasing cost.

$C_{5}$ is the disposal cost per unit.

$\theta t$ is the deterioration rate.

$\mathrm{Q}$ is the order level for quantity.

$T$ is the fixed time horizon.

$t_{1}$ is the decision variable representing the time from which pre-deterioration discount starts.

$\mu$ is the time at which deterioration starts.

$I(t)$ is the inventory level at any time t.

\section{Mathematical Model}

Formulation of the model when pre- and post deterioration discounts on selling price are given is given by Figure-1. At the beginning of the replenishment cycle the inventory level raises to $\mathrm{Q}$. As time progresses inventory level decreases due to stock and time dependent demand up to the time $t_{1}$. At $t_{1}, d_{1} \%$ discount is offered on unit selling price in order to enhance the demand of fresh items. This discount is continued upto the time $\mu$ where deterioration starts. When deterioration starts another $d_{2} \%$ discount is offered on unit selling price to boost the demand. This discount is continued for the rest of the replenishment cycle.

Therefore, the inventory level $I(t)$ at any time $\mathrm{t}$ in the interval[0,T] can be represented by the following differential equations:

$$
\begin{array}{cl}
\frac{d I(t)}{d t}=-(a+b I(t)-c t) & 0 \leq t \leq t_{1} \\
\frac{d I(t)}{d t}=-\alpha_{1}(a+b I(t)-c t) & t_{1} \leq t \leq \mu \\
\frac{d I(t)}{d t}+\theta t I(t)=-\alpha_{2}(a-c t) & \mu \leq t \leq T
\end{array}
$$

The solutions of the above differential equations after applying the boundary conditions $I(0)=Q$ and $I(T)=0$ are given by:

$$
\begin{gathered}
I(t)=\left(Q+\frac{a}{b}+\frac{c}{b^{2}}\right) e^{-b t}+\left(\frac{-a+c t}{b}\right)-\frac{c}{b^{2}} \quad 0 \leq t \leq t_{1} \\
I(t)=-\left(\frac{a-c t}{b}\right)-\frac{c}{b^{2} \alpha_{1}}-\frac{c}{b^{2}}\left(1-\frac{1}{\alpha_{1}}\right) e^{b \alpha_{1}\left(t_{1}-t\right)}+\left(Q+\frac{a}{b}+\frac{c}{b^{2}}\right) e^{b \alpha_{1}\left(t_{1}-t\right)-b t_{1}} \quad t_{1} \leq t \leq \mu \\
I(t)=\alpha_{2}\left[a\left\{(T-\mu)+\frac{\theta(T-\mu)^{3}}{6}-(t-\mu)-\frac{\theta(t-\mu)^{3}}{6}-\frac{\theta t^{2}(T-\mu)}{2}+\frac{\theta t^{2}(t-\mu)}{2}\right\}\right. \\
\left.+c\left\{\frac{(t-\mu)^{2}}{2}+\frac{\theta(t-\mu)^{4}}{8}-\frac{(T-\mu)^{2}}{2}-\frac{\theta(T-\mu)^{4}}{8}-\frac{\theta t^{2}(t-\mu)^{2}}{4}+\frac{\theta t^{2}(T-\mu)^{2}}{4}\right\}\right] \quad \mu \leq t \leq T
\end{gathered}
$$

Order quantity $Q$ is obtained by equating $I(\mu)$ from equations (5) and (6)

$$
Q=\alpha_{2}[X+Y] e^{-b \alpha_{1}\left(t_{1}-\mu\right)+b t_{1}}+Z e^{-b \alpha_{1}\left(t_{1}-\mu\right)+b t_{1}}+M e^{b t_{1}}-\left(\frac{a}{b}+\frac{c}{b^{2}}\right)
$$

Based on above equations, total profit consists of the following elements:

1. Ordering cost during the cycle is 


$$
C_{r}=A
$$

2. Holding cost and disposal cost of inventories during the cycle is

$$
\begin{gathered}
C_{h}+C_{D}=C_{1} \int_{0}^{t_{1}} I(t) d t+C_{1} \int_{t_{1}}^{\mu} I(t) d t+C_{1} \int_{\mu}^{T} I(t) d t+\theta C_{5} \int_{\mu}^{T} t I(t) d t=C_{1}\left[\frac { 1 } { b } ( 1 - e ^ { - b t _ { 1 } } ) \left\{\alpha_{2}\{X+Y\}\right.\right. \\
\left.e^{-b \alpha_{1}\left(t_{1}-\mu\right)+b t_{1}}+Z e^{-b \alpha_{1}\left(t_{1}-\mu\right)+b t_{1}}+M e^{b t_{1}}\right\}-\frac{c t_{1}}{b^{2}}-\frac{a \mu}{b}+\frac{c \mu^{2}}{2 b}-\frac{c\left(\mu-t_{1}\right)}{b^{2} \alpha_{1}}+\frac{1}{\alpha_{1} b} M e^{b \alpha_{1}\left(2 t_{1}-\mu\right)} \\
+\alpha_{2} C_{1}\left[a\left\{\frac{(T-\mu)^{2}}{2}+\frac{\theta}{12}\left(T^{4}+9 \mu^{2} T^{2}-4 \mu^{3} T-6 \mu T^{3}\right)\right\}+c\left\{-\frac{1}{3}(T-\mu)^{3}\right.\right. \\
\left.\left.+\frac{\theta}{120}\left(-8 T^{5}+3 \mu^{5}-40 \mu^{4} T+55 \mu T^{4}+110 \mu^{3} T^{2}-120 \mu^{2} T^{3}\right)\right\}\right] \\
+\alpha_{2} \theta C_{5}\left[a\left\{\frac{1}{6}\left(T^{3}+2 \mu^{3}-3 \mu^{2} T\right)+\frac{\theta}{40}\left(T^{5}-\mu^{5}+10 \mu^{3} T^{2}-5 \mu t^{4}-5 \mu^{4} T\right)\right\}\right. \\
\left.+\frac{\theta}{240}\left(-5 T^{6}-30 \mu^{6}-40 \mu^{3} T^{3}+75 \mu^{4}\left(2 t_{1}-\mu\right)\right]\right] \\
+c\left\{\frac{1}{24}\left(-3 T^{4}+5 \mu^{4}+6 \mu^{2} T^{2}+4 \mu T^{3}-12 \mu^{3} T\right)\right. \\
\left.+\frac{\theta}{5}-30 \mu^{5} T\right)
\end{gathered}
$$

3. Purchase cost in the cycle is given by

$$
\begin{gathered}
C_{P}=C_{4} Q \\
=C_{4} \alpha_{2}(X+Y) e^{-b \alpha_{1}\left(t_{1}-\mu\right)+b t_{1}}+C_{4} Z e^{-b \alpha_{1}\left(t_{1}-\mu\right)+b t_{1}}+C_{4} M e^{b t_{1}}-C_{4}\left(\frac{a}{b}+\frac{c}{b^{2}}\right)
\end{gathered}
$$

4. The total sales revenue in the order cycle is given by

$$
\begin{gathered}
S_{R}=s\left[\int_{0}^{t_{1}}(a+b I(t)-c t) d t+\alpha_{1}\left(1-d_{1}\right) \int_{t_{1}}^{\mu}(a+b I(t)-c t) d t+\alpha_{2}\left(1-d_{2}\right) \int_{\mu}^{T}(a-c t) d t\right] \\
=s\left[\left(1-e^{-b t_{1}}\right)\left\{\alpha_{2}(X+Y) e^{-b \alpha_{1}\left(t_{1}-\mu\right)+b t_{1}}+M e^{b t_{1}}\right\}-\frac{c t_{1}}{b}\right. \\
+\alpha_{1}\left(1-d_{1}\right)\left\{\frac{1}{\alpha_{1}} M e^{b \alpha_{1}\left(2 t_{1}-\mu\right)}-\frac{c\left(\mu-t_{1}\right.}{)} b \alpha_{1}-\frac{1}{\alpha_{1}}\left[\alpha_{2}(X+Y) e^{b \alpha_{1} t_{1}}+Z e^{b \alpha_{1} t_{1}}+M e^{b \alpha_{1}\left(2 t_{1}-\mu\right)}\right]\right\} \\
\left.+\alpha_{2}\left(1-d_{2}\right)\left\{a(T-\mu)-\frac{c}{2}\left(T^{2}-\mu^{2}\right)\right\}\right]
\end{gathered}
$$

Therefore, the total profit per unit time is given by

$$
\begin{gathered}
\operatorname{TPT}\left(d_{2}, t_{1}\right)=\frac{1}{T}\left(S_{R}-C_{P}-C_{h}-C_{D}-C_{r}\right) \\
=\frac{1}{T}\left[\{ s ( 1 - e ^ { - b t _ { 1 } } ) - C _ { 4 } - \frac { C _ { 1 } } { b } ( 1 - e ^ { - b t _ { 1 } } ) \} \left\{\left(1-d_{2}\right)^{-n_{2}}(X+Y) e^{-b\left(1-d_{1}\right)^{-n_{1}\left(t_{1}-\mu\right)+b t_{1}}}\right.\right.
\end{gathered}
$$




$$
\begin{aligned}
& \left.+Z e^{-b\left(1-d_{1}\right)^{-n_{1}}\left(t_{1}-\mu\right)+b t_{1}}+M e^{b t_{1}}\right\}+\left\{s\left(1-d_{1}\right)^{-n_{1}+1}-\frac{C_{1}}{b}\right\} \\
& \left\{\frac{1}{\left(1-d_{1}\right)^{-n_{1}}} M e^{b\left(1-d_{1}\right)^{-n_{1}}\left(2 t_{1}-\mu\right)}-\frac{c\left(\mu-t_{1}\right)}{b\left(1-d_{1}\right)^{-n_{1}}}\right\}-\frac{s c t_{1}}{b}+\frac{c C_{1} t_{1}}{b^{2}}+\frac{a \mu C_{1}}{b}-\frac{c C_{1} \mu^{2}}{2 b} \\
& +\left\{-s\left(1-d_{1}\right)+C_{1}\right\}\left\{\left(1-d_{2}\right)^{-n_{2}}(X+Y) e^{b\left(1-d_{1}\right)^{-n} 1 t_{1}}+Z e^{b\left(1-d_{1}\right)^{-n_{1}} t_{1}}+M e^{b\left(1-d_{1}\right)^{-n_{1}}\left(2 t_{1}-\mu\right)}\right\} \\
& +s\left(1-d_{2}\right)^{-n_{2}+1}\left\{a(T-\mu)-\frac{c}{2}\left(T^{2}-\mu^{2}\right)\right\}+C_{4}\left(\frac{a}{b}+\frac{c}{b^{2}}\right) \\
& -\left(1-d_{2}\right)^{-n_{2}} C_{1}\left[a\left\{\frac{(T-\mu)^{2}}{2}+\frac{\theta}{12}\left(T^{4}+9 \mu^{2} T^{2}-4 \mu^{3} T-6 \mu T^{3}\right)\right\}+c\left\{-\frac{1}{3}(T-\mu)^{3}\right.\right. \\
& \left.\left.+\frac{\theta}{120}\left(-8 T^{5}+3 \mu^{5}-40 \mu^{4} T+55 \mu T^{4}+110 \mu^{3} T^{2}-120 \mu^{2} T^{3}\right)\right\}\right] \\
& -\left(1-d_{2}\right)^{-n_{2}} \theta C_{5}\left[a\left\{\frac{1}{6}\left(T^{3}+2 \mu^{3}-3 \mu^{2} T\right)+\frac{\theta}{40}\left(T^{5}-\mu^{5}+10 \mu^{3} T^{2}-5 \mu T^{4}-5 \mu^{4} T\right)\right\}\right. \\
& +c\left\{\frac{1}{24}\left(-3 T^{4}+5 \mu^{4}+6 \mu^{2} T^{2}+4 \mu T^{3}-12 \mu^{3} T\right)\right. \\
& \left.\left.\left.+\frac{\theta}{240}\left(-5 T^{6}-30 \mu^{6}-40 \mu^{3} T^{3}+75 \mu^{4} T^{2}+30 \mu T^{5}-30 \mu^{5} T\right)\right\}\right]-A\right]
\end{aligned}
$$

It is to be noted that here the two discounts $d_{1}$ and $d_{2}$ are given on constant unit selling prices of the product. There may be another case if the discount $d_{2}$ after the start of deterioration may be given on the pre-deterioration discounted selling price $\left(1-d_{1}\right) s$.

The pre-deterioration discount on selling price is to be given in such a way that the discounted selling price is not less than the unit cost of the product, i.e. $s\left(1-d_{1}\right)-c>0$.

\section{Solution Procedure}

According to equation (12), TPT $\left(d_{2}, t_{1}\right)$ is a function of $d_{2}$ and $t_{1}$. To maximize total profit per unit timeT PT $\left(d_{2}, t_{1}\right)$, the optimal values of $d_{2}$ and $t_{1}$ are obtained by solving the following equations simultaneously.

$$
\frac{\partial T P T\left(d_{2} t_{1}\right)}{\partial d_{2}}=0
$$

And

$$
\frac{\partial T P T\left(d_{2}, t_{1}\right)}{\partial t_{1}}=0
$$

Provided

$$
\left(\frac{\partial^{2} T P T}{\partial d_{2}^{2}}\right)\left(\frac{\partial^{2} T P T}{\partial t_{1}^{2}}\right)-\left(\frac{\partial^{2} T P T}{\partial d_{2} \partial t_{1}}\right)^{2}<0
$$

Equation (13) and (14) are equivalent to

i.e.

$$
\begin{aligned}
\frac{1}{T}\left[\left\{s\left(1-e^{-b t_{1}}\right)-C_{4}-\frac{C_{1}}{b}\left(1-e^{-b t_{1}}\right)\right\}\left\{n_{2}\left(1-d_{2}\right)^{-n_{2}-1}(X+Y) e^{-b\left(1-d_{1}\right)^{-n_{1}}\left(t_{1}-\mu\right)+b t_{1}}\right\}\right. \\
+\left\{-s\left(1-d_{1}\right)+C_{1}\right\}\left\{n_{2}\left(1-d_{2}\right)^{-n_{2}-1}(X+Y) e^{b\left(1-d_{1}\right)^{-n_{1}} t_{1}}\right\} \\
-s\left(1-n_{2}\right)\left(1-d_{2}\right)^{-n_{2}}\left\{a(T-\mu)-\frac{c}{2}\left(T^{2}-\mu^{2}\right)\right\} \\
-n_{2}\left(1-d_{2}\right)^{-n_{2}-1} C_{1}\left[a\left\{\frac{(T-\mu)^{2}}{2}+\frac{\theta}{12}\left(T^{4}+9 \mu^{2} T^{2}-4 \mu^{3} T-6 \mu T^{3}\right)\right\}+c\left\{-\frac{1}{3}(T-\mu)^{3}\right.\right.
\end{aligned}
$$




$$
\begin{gathered}
\left.\left.+\frac{\theta}{120}\left(-8 T^{5}+3 \mu^{5}-40 \mu^{4} T+55 \mu T^{4}+110 \mu^{3} T^{2}-120 \mu^{2} T^{3}\right)\right\}\right] \\
-n_{2}\left(1-d_{2}\right)^{-n_{2}-1} \theta C_{5}\left[a\left\{\frac{1}{6}\left(T^{3}+2 \mu^{3}-3 \mu^{2} T\right)+\frac{\theta}{40}\left(T^{5}-\mu^{5}+10 \mu^{3} T^{2}-5 \mu T^{4}-5 \mu^{4} T\right)\right\}\right. \\
+c\left\{\frac{1}{24}\left(-3 T^{4}+5 \mu^{4}+6 \mu^{2} T^{2}+4 \mu T^{3}-12 \mu^{3} T\right)\right. \\
\left.\left.\left.+\frac{\theta}{240}\left(-5 T^{6}-30 \mu^{6}-40 \mu^{3} T^{3}+75 \mu^{4} T^{2}+30 \mu T^{5}-30 \mu^{5} T\right)\right\}\right]-A\right]=0
\end{gathered}
$$

And

$$
\begin{aligned}
& \frac{1}{T}\left[\left\{s b e^{-b t_{1}}-C_{1} e^{-b t_{1}}\right\}\left\{\left(1-d_{2}\right)^{-n_{2}}(X+Y) e^{-b\left(1-d_{1}\right)^{-n_{1}}\left(t_{1}-\mu\right)+b t_{1}}+Z e^{-b\left(1-d_{1}\right)^{-n_{1}}\left(t_{1}-\mu\right)+b t_{1}}+M e^{b t_{1}}\right\}\right. \\
& +\left\{s\left(1-e^{-b t_{1}}\right)-C_{4}-\frac{C_{1}}{b}\left(1-e^{-b t_{1}}\right)\right\}\left\{b\left(1-\left(1-d_{1}\right)^{-n_{1}}\right)\left(1-d_{2}\right)^{-n_{2}}(X+Y) e^{-b\left(1-d_{1}\right)^{-n_{1}}\left(t_{1}-\mu\right)+b t_{1}}\right. \\
& \left.+b\left(1-\left(1-d_{1}\right)^{-n_{1}}\right) Z e^{-b\left(1-d_{1}\right)^{-n_{1}}\left(t_{1}-\mu\right)+b t_{1}}+b M e^{b t_{1}}\right\}+\left\{s\left(1-d_{1}\right)^{-n_{1}+1}-\frac{C_{1}}{b}\right\} \\
& \left\{2 b M e^{b\left(1-d_{1}\right)^{-n_{1}}\left(2 t_{1}-\mu\right)}+\frac{c}{b\left(1-d_{1}\right)^{-n_{1}}}\right\}-\frac{s c}{b}+\frac{c C_{1}}{b^{2}} \\
& +\left\{-s\left(1-d_{1}\right)+C_{1}\right\}\left\{b\left(1-d_{1}\right)^{-n_{1}}\left(1-d_{2}\right)^{-n_{2}}(X+Y) e^{b\left(1-d_{1}\right)^{-n_{1}} t_{1}}+\left(1-d_{1}\right)^{-n_{1}} b Z\right. \\
& \left.\left.e^{b\left(1-d_{1}\right)^{-n_{1}} t_{1}}+2 b\left(1-d_{1}\right)^{-n_{1}} M e^{b\left(1-d_{1}\right)^{-n_{1}}\left(2 t_{1}-\mu\right)}\right\}\right]=0
\end{aligned}
$$

Where $X=a(T-\mu)\left\{1-\frac{\theta \mu^{2}}{2}+\frac{\theta(T-\mu)^{2}}{6}\right\}, Y=c \frac{(T-\mu)^{2}}{2}\left\{-1+\frac{\theta \mu^{2}}{2}-\frac{\theta(T-\mu)^{2}}{4}\right\}, Z=\left\{\frac{a-c \mu}{b}+\frac{c}{b^{2}\left(1-d_{1}\right)^{-n_{1}}}\right\}$ and $M=\frac{c}{b^{2}}\left(1-\frac{1}{\left(1-d_{1}\right)^{-n_{1}}}\right)$

\section{Special Cases}

\section{CASE 1:}

If $c=0$, then the demand rate reduces to $D(t)=a+b I(t)$ i.e. the demand becomes stock dependent.

$$
\begin{array}{cl}
\frac{d I(t)}{d t}=-(a+b I(t)) & 0 \leq t \leq t_{1} \\
\frac{d I(t)}{d t}=-\alpha_{1}(a+b I(t)) & t_{1} \leq t \leq \mu \\
\frac{d I(t)}{d t}+\theta t I(t)=-\alpha_{2} a & \mu \leq t \leq T
\end{array}
$$

The solutions of the above differential equations after applying the boundary conditions $I(0)=Q$ and $I(T)=0$ are given by:

$$
\begin{gathered}
I(t)=\left(Q+\frac{a}{b}\right) e^{-b t}-\frac{a}{b} \quad 0 \leq t \leq t_{1} \\
I(t)=-\frac{a}{b}+\left(Q+\frac{a}{b}\right) e^{b \alpha_{1}\left(t_{1}-t\right)-b t_{1}} \\
I(t)=\alpha_{2}\left[a\left\{(T-\mu)+\frac{\theta(T-\mu)^{3}}{6}-(t-\mu)-\frac{\theta(t-\mu)^{3}}{6}-\frac{\theta t^{2}(T-\mu)}{2}+\frac{\theta t^{2}(t-\mu)}{2}\right\}\right]
\end{gathered}
$$

Order quantity for pre- and post deterioration discount on unit selling price is given by 


$$
Q=\alpha_{2} X e^{-b \alpha_{1}\left(t_{1}-\mu\right)+b t_{1}}+\frac{a}{b}\left\{e^{-b \alpha_{1}\left(t_{1}-\mu\right)+b t_{1}}-1\right\}
$$

The total profit per unit time is given by

$$
\begin{gathered}
T P T\left(d_{2}, t_{1}\right)=\frac{1}{T}\left[\{ s ( 1 - e ^ { - b t _ { 1 } } ) - C _ { 4 } - \frac { C _ { 1 } } { b } ( 1 - e ^ { - b t _ { 1 } } ) \} \left\{\left(1-d_{2}\right)^{-n_{2}} X e^{-b\left(1-d_{1}\right)^{-n_{1}}\left(t_{1}-\mu\right)+b t_{1}}\right.\right. \\
\left.+\frac{a}{b} e^{-b\left(1-d_{1}\right)^{-n_{1}}\left(t_{1}-\mu\right)+b t_{1}}\right\}+\frac{a \mu C_{1}}{b}+\left\{-s\left(1-d_{1}\right)+C_{1}\right\}\left\{\left(1-d_{2}\right)^{-n_{2}} X e^{b\left(1-d_{1}\right)^{-n} t_{1}}+\frac{a}{b} e^{b\left(1-d_{1}\right)^{-n_{1} t_{1}}}\right\} \\
+s\left(1-d_{2}\right)^{-n_{2}+1} a(T-\mu)+C_{4} \frac{a}{b}-\left(1-d_{2}\right)^{-n_{2}} C_{1} a\left\{\frac{(T-\mu)^{2}}{2}+\frac{\theta}{12}\left(T^{4}+9 \mu^{2} T^{2}-4 \mu^{3} T-6 \mu T^{3}\right)\right\} \\
\left.-\left(1-d_{2}\right)^{-n_{2}} \theta C_{5} a\left\{\frac{1}{6}\left(T^{3}+2 \mu^{3}-3 \mu^{2} T\right)+\frac{\theta}{40}\left(T^{5}-\mu^{5}+10 \mu^{3} T^{2}-5 \mu T^{4}-5 \mu^{4} T\right)\right\}-A\right]
\end{gathered}
$$

$T P T\left(d_{2}, t_{1}\right)$ is a function of $d_{2}$, and $t_{1}$. To maximize total profit per unit timeT PT $\left(d_{2}, t_{1}\right)$, the optimal values of $d_{2}$ and $t_{1}$ can be obtained by solving the following equations simultaneously.

$$
\frac{\partial T P T\left(d_{2}, t_{1}\right)}{\partial d_{2}}=0
$$

And

$$
\frac{\partial T P T\left(d_{2}, t_{1}\right)}{\partial t_{1}}=0
$$

Provided

$$
\left(\frac{\partial^{2} T P T}{\partial d_{2}^{2}}\right)\left(\frac{\partial^{2} T P T}{\partial t_{1}^{2}}\right)-\left(\frac{\partial^{2} T P T}{\partial d_{2} \partial t_{1}}\right)^{2}<0
$$

Equations (24) and (25) are equivalent to

$$
\begin{gathered}
\frac{1}{T}\left[\left\{s\left(1-e^{-b t_{1}}\right)-C_{4}-\frac{C_{1}}{b}\left(1-e^{-b t_{1}}\right)\right\}\left\{n_{2}\left(1-d_{2}\right)^{-n_{2}-1} X e^{-b\left(1-d_{1}\right)^{-n_{1}}\left(t_{1}-\mu\right)+b t_{1}}\right\}\right. \\
+\left\{-s\left(1-d_{1}\right)+C_{1}\right\}\left\{n_{2}\left(1-d_{2}\right)^{-n_{2}-1} X e^{b\left(1-d_{1}\right)^{-n_{1}} t_{1}}\right\}-s\left(1-n_{2}\right)\left(1-d_{2}\right)^{-n_{2}} a(T-\mu) \\
-n_{2}\left(1-d_{2}\right)^{-n_{2}-1} C_{1} a\left\{\frac{(T-\mu)^{2}}{2}+\frac{\theta}{12}\left(T^{4}+9 \mu^{2} T^{2}-4 \mu^{3} T-6 \mu T^{3}\right)\right\} \\
\left.-n_{2}\left(1-d_{2}\right)^{-n_{2}-1} \theta C_{5} a\left\{\frac{1}{6}\left(T^{3}+2 \mu^{3}-3 \mu^{2} T\right)+\frac{\theta}{40}\left(T^{5}-\mu^{5}+10 \mu^{3} T^{2}-5 \mu T^{4}-5 \mu^{4} T\right)\right\}\right]=0 \\
\quad \frac{1}{T}\left[\left\{s b e^{-b t_{1}}-C_{1} e^{-b t_{1}}\right\}\left\{\left(1-d_{2}\right)^{-n_{2}} X e^{-b\left(1-d_{1}\right)^{-n_{1}}\left(t_{1}-\mu\right)+b t_{1}}+\frac{a}{b} e^{-b\left(1-d_{1}\right)^{-n_{1}}\left(t_{1}-\mu\right)+b t_{1}}\right\}\right. \\
+\left\{s\left(1-e^{-b t_{1}}\right)-C_{4}-\frac{C_{1}}{b}\left(1-e^{-b t_{1}}\right)\right\}\left\{b\left(1-\left(1-d_{1}\right)^{-n_{1}}\right)\left(1-d_{2}\right)^{-n_{2}} X e^{-b\left(1-d_{1}\right)^{-n_{1}}\left(t_{1}-\mu\right)+b t_{1}}\right. \\
\left.+\left(1-\left(1-d_{1}\right)^{-n_{1}}\right) a e^{-b\left(1-d_{1}\right)^{-n_{1}}\left(t_{1}-\mu\right)+b t_{1}}\right\}+\left\{-s\left(1-d_{1}\right)+C_{1}\right\}\left\{b\left(1-d_{1}\right)^{-n_{1}}\left(1-d_{2}\right)^{-n_{2}} X e^{b\left(1-d_{1}\right)^{-n_{1}} t_{1}}\right. \\
\left.\left.+\left(1-d_{1}\right)^{-n_{1}} a e^{b\left(1-d_{1}\right)^{-n_{1}} t_{1}}\right\}\right]=0
\end{gathered}
$$

CASE 2: Model for fixed life time products

If the product has a fixed shelf life then the post deterioration discount does not comes into account and only pre- deterioration discount on selling price is given. So, $\mu=T, d_{2}=0$.

Hence, from equation (7) the initial inventory level is given as:

$$
Q=\left\{\frac{a-c T}{b}+\frac{c}{b^{2} \alpha_{1}}\right\} e^{-b \alpha_{1}\left(t_{1}-T\right)+b t_{1}}+M e^{b t_{1}}-\left(\frac{a}{b}+\frac{c}{b^{2}}\right)
$$


and the total profit per unit time $T P T\left(t_{1}\right)$ over the period $[0, \mathrm{~T}]$ is reduced to

$$
\begin{gathered}
T P T\left(t_{1}\right)=\frac{1}{T}\left[\{ s ( 1 - e ^ { - b t _ { 1 } } ) - C _ { 4 } - \frac { C _ { 1 } } { b } ( 1 - e ^ { - b t _ { 1 } } ) \} \left\{\left\{\frac{a-c T}{b}+\frac{c}{b^{2}\left(1-d_{1}\right)^{-n_{1}}}\right\} e^{-b\left(1-d_{1}\right)^{-n_{1}\left(t_{1}-T\right)+b t_{1}}}\right.\right. \\
\left.+M e^{b t_{1}}\right\}+\left\{s\left(1-d_{1}\right)^{-n_{1}+1}-\frac{C_{1}}{b}\right\}\left\{\frac{1}{\left(1-d_{1}\right)^{-n_{1}}} M e^{b\left(1-d_{1}\right)^{-n_{1}}\left(2 t_{1}-T\right)}-\frac{c\left(T-t_{1}\right)}{b\left(1-d_{1}\right)^{-n_{1}}}\right\}-\frac{s c t_{1}}{b}+\frac{c C_{1} t_{1}}{b^{2}}+\frac{a T C_{1}}{b}-\frac{c C_{1} T^{2}}{2 b} \\
+\left\{-s\left(1-d_{1}\right)+C_{1}\right\}\left\{\left\{\frac{a-c T}{b}+\frac{c}{b^{2}\left(1-d_{1}\right)^{-n_{1}}}\right\} e^{b\left(1-d_{1}\right)^{-n_{1} t_{1}}}+M e^{\left.b\left(1-d_{1}\right)^{-n_{1}\left(2 t_{1}-T\right)}\right\}}\right. \\
\left.+C_{4}\left(\frac{a}{b}+\frac{c}{b^{2}}\right)-A\right]
\end{gathered}
$$

To maximize total profit per unit timeT PT $\left(t_{1}\right)$, the optimal value of $t_{1}$ can be obtained by solving the following differential equation.

$$
\frac{\partial T P T}{\partial t_{1}}=0
$$

Provided

$$
\begin{gathered}
\frac{\partial^{2} T P T}{\partial t_{1}^{2}}<0 \\
\frac{1}{T}\left[\left\{s b e^{-b t_{1}}-C_{1} e^{-b t_{1}}\right\}\left\{\left\{\frac{a-c T}{b}+\frac{c}{b^{2}\left(1-d_{1}\right)^{-n_{1}}}\right\} e^{-b\left(1-d_{1}\right)^{-n_{1}}\left(t_{1}-T\right)+b t_{1}}+M e^{b t_{1}}\right\}\right. \\
+\left\{s\left(1-e^{-b t_{1}}\right)-C_{4}-\frac{C_{1}}{b}\left(1-e^{-b t_{1}}\right)\right\}\left\{\left(1-\left(1-d_{1}\right)^{-n_{1}}\right)\left\{a-c T+\frac{c}{b\left(1-d_{1}\right)^{-n_{1}}}\right\} e^{-b\left(1-d_{1}\right)^{-n_{1}}\left(t_{1}-T\right)+b t_{1}}\right. \\
\left.+M e^{b t_{1}}\right\}+\left\{s\left(1-d_{1}\right)^{-n_{1}+1}-\frac{C_{1}}{b}\right\}\left\{2 b M e^{b\left(1-d_{1}\right)^{-n_{1}}\left(2 t_{1}-T\right)}+\frac{c}{b\left(1-d_{1}\right)^{-n_{1}}}\right\}-\frac{s c}{b}+\frac{c C_{1}}{b^{2}} \\
\left.+\left\{-s\left(1-d_{1}\right)+C_{1}\right\}\left\{\left(1-d_{1}\right)^{-n_{1}}\left\{a-c T+\frac{c}{b\left(1-d_{1}\right)^{-n_{1}}}\right\} e^{b\left(1-d_{1}\right)^{-n_{1}}} t_{t_{1}}+2 b\left(1-d_{1}\right)^{-n_{1}} M e^{b\left(1-d_{1}\right)^{-n_{1}}\left(2 t_{1}-T\right)}\right\}\right]=0
\end{gathered}
$$

\section{Numerical Examples}

\section{Example 1:}

When both pre- and post deterioration discounts on unit selling price are given, a practical model is considered taking the following values for different parameters: $a=110$ units, $b=0.5, c=0.35, T=10$ months, $s=$ Rs. $12, \theta=0.009, n_{1}=1$, $n_{2}=2, \quad \mu=5.5, \quad A=$ Rs. 300 per order, $C_{1}=0.75, C_{4}=6, C_{5}=0.60, d_{1}=0.3$ Using the solution procedure described in the model the optimal results obtained are, $d_{2}^{*}=0.573715, t_{1}^{*}=2.5424, T P T\left(d_{2}^{*}, t_{1}^{*}\right)=$ Rs. 843.373 and $Q^{*}=77473.3$. Thus the pre-deterioration discount of $30 \%$ on unit selling price starts at time $t_{1}^{*}=2.5424$ and when product start to deteriorate $57.37 \%$ discount on unit selling price is given for the remaining period of replenishment cycle in order to obtain the maximum profit $T P T\left(d_{2}^{*}, t_{1}^{*}\right)=$ Rs. 843.373 on optimal order quantity $Q^{*}=77473.3$.

Example 2: When $c=0$, then the demand rate reduces to $D(t)=a+b I(t)$ i.e. the demand becomes only stock dependent. Using the parameters of Example- 1 the results obtained are, $d_{2}^{*}=0.580829, t_{1}^{*}=2.54243, T P T\left(d_{2}^{*}, t_{1}^{*}\right)=$ Rs. 875.159 and $Q^{*}=80539.1$. Thus the pre-deterioration discount of $30 \%$ on unit selling price starts at time $t_{1}^{*}=2.54243$ and when product start to deteriorate $58.08 \%$ discount on unit selling price is given for the remaining period of replenishment cycle in order to obtain the maximum profit $\operatorname{TPT}\left(d_{2}^{*}, t_{1}^{*}\right)=$ Rs. 875.159 on optimal order quantity $Q *=80539.1$.

Example 3: Based on Case 2, When products have fixed lifetime, only pre-deterioration discounts on unit selling price is given, a practical model is considered taking the following values for different parameters: $a=110, b=0.5, c=0.35$, $T=5.5, s=12, \theta=0.009, n_{1}=1, n_{2}=2, \quad \mu=5.5, \quad A=300, \quad C_{1}=0.75, \quad C_{4}=6, \quad C_{5}=0.60, \quad d_{1}=0.3$

Using the solution procedure described in the model the results obtained are, $t_{1}^{*}=2.5424, T P T\left(t_{1}^{*}\right)=$ Rs. 297.885 and $Q^{*}=$ 6181.32. Thus in order to enhance inventory depletion rate the pre-deterioration discount of $30 \%$ on unit selling price should start at time $t_{1}=2.54251$ when product has a fixed lifetime period. 


\section{Sensitivity Analysis}

To study the effect of changes of the parameters on the optimal profit derived by proposed method, a sensitivity analysis is performed considering the numerical examples given above. Sensitivity analysis is performed by changing (increasing or decreasing) the parameters by $20 \% \& 50 \%$ and taking one parameter at a time, keeping the remaining parameters at their original values. The results are shown in Table 1 for Example 1

A careful study of Table 1 reveals the following:

i. $d_{2}$ is slightly sensitive to changes in the values of parameters $c, C_{5} \& a$, it is moderately sensitive to changes in $\theta$ and neutral to changes in $A$.

ii. $t_{1}$ is neutral to changes in $c, C_{5}, a, \theta \& A$.

iii. $Q$ is slightly sensitive to changes in the values of parameters $c, C_{5} \& \theta$, it is highly sensitive to change in $a$ and neutral to changes in $A$.

iv. $T P T$ is slightly sensitive to changes in the values of parameters $c \& C_{5}$, it is moderately sensitive to changes in $\theta \& A$ and highly sensitive to change in a.

\section{Conclusion}

In this paper an inventory model is developed with stock dependent and time varying decreasing demand. Deterioration is non-instantaneous \& time dependent. After some time when product starts to deteriorate the demand becomes stock independent and is only time decreasing. A mathematical model is developed incorporating both pre-and post deterioration discounts on unit selling price. Optimal discount on unit selling price and optimal time to give the discount for maximizing the total profit per unit are determined. Results obtained imply that the effect of pre-and post deterioration discount and non-instantaneous time dependent deterioration on the total profit is more significant than a policy which ignores the effects of these factors. An analytic formulation of the problem on the frame work described above and optimal solution procedure to find optimal discount is presented. Sensitivity analysis with respect to various parameters has been carried out.

Thus this model incorporates some realistic features that are likely to be associated with some kind of inventory. The model could be very useful in retail business. It can be used for electronic components, fashionable goods, clothes, foodstuffs (vegetables and fruits) and other products which have more likely the characteristics above.

The present study can be further extended for some other factors involved in the inventory system.

\section{References}

Ardalan, A. (1994). Optimal prices \& Order quantities when temporary price discounts result on increase in demand, European Journal of Operational Research, 72(1), 52-61.

Balkhi, Z. T. and Benkheronf, L. (2004). On an inventory model for deteriorating items with stock dependent and time varying demand rates, Computers \& Operations Research, 31(2), 223-240.

Banerjee, S. and Sharma, A. (2010). Optimal procurement and pricing policy for inventory models with price and time dependent seasonal demand, Mathematical and Computer Modeling, 51, 700-714.

Benkheronf, L. (1995). Theory \& Methodology on an inventory model with deteriorating item and decreasing timevarying demand and shortages, European Journal of Operational Research, 86, 293-299.

Cardenas-Barron, L. E, Smith, N. R. and Goyal, S. K. (2010). Optimal Order size to take advantage of a one-time discount offer with allowed backorders, Applied Mathematical Modeling, 34, 1642-1652.

Castro, I. T. and Alfa, A. S. (2004). Lifetime replacement policy in discrete time for a single init system, Reliability Engineering \& System Safety, 84, 103-111.

Chang, C. T., Teng, J. T. and Goyal, S. K. (2010). Optimal replenishment policies for non-instantaneous deteriorating items with stock-dependent demand, International Journal of Production Economics, 123, 62-68.

Chung, K. J., Chu, P., Lan, S. P. (2000). A note on EOQ models for deteriorating items under stock dependent selling rate, European Journal of Operational Research, 124, 550-559.

Cobbaert, K. and Oudheusden, D. V. (1996). Inventory models for fast moving spare parts subject to "sudden death" obsolescence, International Journal of Production Economics, 44, 239-248.

Ghare, P. M. and Schrader, G. F. (1963). A model for exponentially decaying inventory system, International Journal of Production Research, 21,449-460.

Goyal, S. K. and Giri, B. C. (2001). Recent trends in modeling of deteriorating inventory, European Journal of Operational Research, 134, 1-16. 
Hsu, W. K. and Yu, H. F. (2009). An EOQ model for imperfective items under a onetime only discount, Omega, 37(5), 1018-1026.

Lui, L and Shi, D. (1999). An (S,S) model for inventory with exponential lifetimes and renewal demands, Naval research Logistic, 46, 39-56.

Moon, I., Giri, B.C. and Ko, B. (2005). Economic Order Quantity models for ameliorating/ deteriorating items under inflation \& time discounting, European Journal of Operational Research, 162, 773-785.

Nahmias, P. (1982). Perishable inventory theory; a review, Operations Research, 30, 680-708.

Omar, M. (2009). An integrated equal-lots policy for shipping a vendor's final production batch to a single buyer under linearly decreasing demand, International Journal of Production Economics, 118, 185-188.

Ouyang, L. Y., Wu, K. S. and Yang, C. T. (2006). A study on an inventory model for non-instantaneous deteriorating items with permissible delay in payments, Computer \& Industrial Engineering, 51, 637-651.

Panda, S., Saha, S. and Basu, M. (2009). An EOQ model for perishable products with discounted selling price and stock dependent demand, Central European Journal of Operational Research, 17(1), 31-53.

Papachristos, S. and Skouri, K. (2003). An inventory model with deteriorating items, quantity discount, pricing and time-dependent partial backlogging, International Journal of Production Economics, 83, 247-256.

Sana, S. S. and Chaudheeri, K. S. (2008). A deterministic EOQ models with delays in payments and price-discount offers. European Journal of Operational Research, 184, 509-533.

Smith, P. H. (1977). Optimal production policies for items with decreasing demand, European Journal of Operational Research, 1(6), 365-367.

Wee, H. M. and Yu, J. (1997). A deteriorating inventory model with a temporary price discount, International Journal of Production Economics, 53, 81-90

Wu, K. S., Ouyang, L. Y. and yang, C. T. (2006). An optimal replenishment policy for non-instantaneous deteriorating items with stock-dependent demand \& partial backlogging, International Journal of Production Economics, 101, 369-384.

Yang, J., Zhao, G. R. and rand, G. K. (2004). An eclectic approach for replenishment with non-liner decreasing demand, International Journal of Production Economics, 92, 125-131.

Zhao, G. Q., Yang, J. and Rand, G. K. (2001). Heuristics for replenishment with linear decreasing demand, International Journal of Production Economics, 69, 339-345.

Zhou, Y. W. (2003). A multi-warehouse inventory model for items with time-varying demand and shortages, Computers \& Operations Research, 30(14), 2115-2134. 
Table 1.

\begin{tabular}{|l|l|l|l|l|l|}
\hline Parameter & \% change & \% change in $d_{2}$ & \% change in $t_{1}$ & \% change in TPT & \% change in Q \\
\hline $\mathrm{c}$ & -50 & 0.0063 & 0.0000 & 0.0188 & 0.0197 \\
\hline & -20 & 0.0025 & 0.0000 & 0.0075 & 0.0079 \\
\hline & 20 & -0.0026 & 0.0000 & -0.0075 & -0.0079 \\
\hline & 50 & -0.0064 & 0.0000 & -0.0187 & -0.0196 \\
\hline & -50 & -0.0482 & 0.0000 & -0.0491 & -0.0661 \\
\hline & -20 & -0.0243 & 0.0000 & -0.0255 & -0.0347 \\
\hline & -20 & 0.0248 & 0.0000 & 0.0278 & 0.0386 \\
\hline$C_{5}$ & -20 & 0.0627 & 0.0000 & 0.0743 & 0.1053 \\
\hline & -50 & 0.0104 & 0.0000 & 0.0114 & 0.0262 \\
\hline & -20 & 0.0041 & 0.0000 & 0.0045 & 0.0103 \\
\hline & 20 & -0.0041 & 0.0000 & -0.0045 & -0.0102 \\
\hline $\mathrm{a}$ & 50 & -0.0104 & 0.0000 & -0.0111 & -0.0251 \\
\hline & -50 & -0.0130 & 0.0000 & -0.5364 & -0.5195 \\
\hline & -20 & -0.0032 & 0.0000 & -0.2146 & -0.2079 \\
\hline & 20 & 0.0021 & 0.0000 & 0.2146 & 0.2079 \\
\hline A & 50 & 0.0042 & 0.0000 & 0.5366 & 0.5197 \\
\hline & -50 & 0.0000 & 0.0000 & 0.0178 & 0.0000 \\
\hline & -20 & 0.0000 & 0.0000 & 0.0071 & 0.0000 \\
\hline & 20 & 0.0000 & 0.0000 & -0.0071 & 0.0000 \\
\hline & 50 & 0.0000 & 0.0000 & -0.0178 & 0.0000 \\
\hline
\end{tabular}

-....

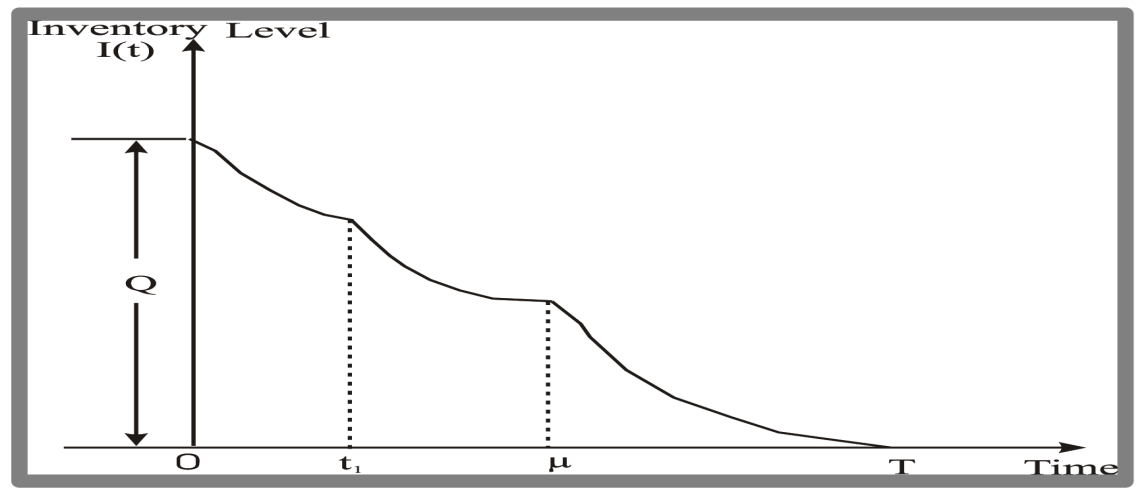

Figure 1. 